\title{
Médiévales
}

Langues, Textes, Histoire

54 | printemps 2008

Frères et sœurs

\section{Liens adelphiques et endogamie géographique dans les Marches de la première moitié du XIV ${ }^{\mathrm{e}}$ siècle}

Sibship links and geographical endogamy in early fourteenth-century Marche.

\section{Didier Lett}

\section{OpenEdition}

\section{Journals}

Édition électronique

URL : https://journals.openedition.org/medievales/4742

DOI : $10.4000 /$ medievales.4742

ISSN : 1777-5892

\section{Éditeur}

Presses universitaires de Vincennes

\section{Édition imprimée}

Date de publication : 1 juin 2008

Pagination : 53-68

ISBN : 978-2-84292-217-7

ISSN : 0751-2708

\section{Référence électronique}

Didier Lett, «Liens adelphiques et endogamie géographique dans les Marches de la première moitié du xIv ${ }^{e}$ siècle », Médiévales [En ligne], 54 | printemps 2008, mis en ligne le 10 septembre 2010, consulté le 22 avril 2022. URL : http://journals.openedition.org/medievales/4742 ; DOI : https://doi.org/

10.4000 /medievales.4742

Ce document a été généré automatiquement le 22 avril 2022.

Tous droits réservés 


\title{
Liens adelphiques et endogamie géographique dans les Marches de la première moitié du XIV siècle
}

Sibship links and geographical endogamy in early fourteenth-century Marche.

\author{
Didier Lett
}

1 La proximité spatiale est un élément déterminant dans la construction de la parentèle car les sentiments sont conditionnés par le partage quotidien d'expériences, des intérêts communs et des échanges ${ }^{1}$. L'étude de la parenté et, plus généralement, du lien social, au Moyen Âge doit prendre davantage en compte le fait de vivre sous le même toit, d'appartenir à un même micro-espace (même groupe de maison, même quartier, même village) et analyser les conséquences pratiques de cette familiarité. Or, au cours de son existence, un individu ne partage pas son espace de vie avec les mêmes personnes, apparentées ou non. Les femmes, plus que les hommes, sont amenées à se déplacer car, dans la grande majorité des cas, à la suite du mariage, elles quittent la maison de leur père pour celle de leur époux. Cette délocalisation féminine se manifeste à des échelles variables dans l'espace et dans le temps ${ }^{2}$. Un des enjeux centraux, pour qui s'intéresse à l'histoire de la famille et de la parenté, est de tenter de mesurer la capacité de résistance des liens de parenté (de filiation, d'alliance et de germanité), à l'éloignement géographique ${ }^{3}$.

Dans cette optique, nous voudrions ici tenter de mesurer les incidences de la pratique du mariage virilocal ou patrilocal sur les relations entre frères et sœurs en nous appuyant, pour l'essentiel, sur le procès de canonisation de Nicolas de Tolentino (1325), documentation qui permet, comme l'ensemble des sources hagiographiques, non seulement de construire l'histoire des pratiques religieuses mais également d'élaborer une histoire sociale ${ }^{4}$. Lors du trauma que représente la maladie, l'accident, la mort ou la perception d'un miracle, les témoignages permettent de saisir auprès d'ego, la parenté disponible, soit que cette dernière ait été « réellement » convoquée à tel ou tel moment de son cycle de vie, soit que ce lien ait resurgi à la mémoire du déposant face aux 
commissaires qui mènent l'interrogatoire, soit que le témoin ait cru utile et nécessaire de l'évoquer dans le cadre de la procédure inquisitoire pour faire preuve.

Suivant les voies tracées par l'anthropologie historique, la plupart des travaux sur l'exogamie ou l'endogamie géographique dans l'espace italien médiéval (à notre connaissance il n'en n'existe pas pour les Marches de la fin du Moyen Âge) s'intéressent avant tout, à mesurer la manière dont les familles étendent (socialement et géographiquement) l'alliance en dehors des cercles plus familiers de la consanguinité et du voisinage sans s'interroger sur les conséquences pratiques de la création de ces nouvelles relations ${ }^{5}$.

Nous partirons de l'analyse ethnographique d'un cas, tel qu'il apparaît dans un des témoignages du procès de canonisation de Nicolas de Tolentino, pour montrer la forte présence des frères et des sœurs auprès du témoin et/ou du miraculé. On constatera ensuite, qu'essentiellement à cause de la prégnance du mariage virilocal dans les Marches du début du XIV ${ }^{e}$ siècle, les frères et les sœurs du mari sont davantage présents que ceux de l'épouse mais que les liens adelphiques de la femme résistent, en partie à cause d'une délocalisation à faible distance.

Les fratries d'Alixa

Le 10 septembre 1325 au matin, Frédéric, évêque de Senigalia, et Thomas, évêque de Cesena, accompagnés de deux notaires, s'installent dans le couvent des Augustins de Tolentino, quasi-città des Marches, peuplée d'environ 6000 habitants, appartenant au diocèse de Camerino, dans la Marche d'Ancône. Ils se tiennent prêts à entendre et à consigner de nouveaux témoignages sur la fama sanctitatis et les miracles qu'un ermite de saint Augustin, Nicolas de Tolentino, mort vingt ans auparavant, aurait accomplis. Mandatés par le pape Jean XXII qui, sous la pression des Augustins et des notables de Tolentino, a conféré l'autorisation apostolique de compiler un procès en vue de la canonisation de Nicolas (par une bulle datée du 23 mai 1325), Thomas et Frédéric ont commencé leurs interrogatoires près de deux mois auparavant à Tolentino, puis se sont déplacés à San Ginesio, Camerino et San Severino, avant de revenir dans la ville de Nicolas (les 9 et 10 septembre) et de terminer leur pérégrination le 28 septembre à Macerata, siège du recteur de la Marche d'Ancône (voir carte en annexe). Au total, ils entendent de très nombreux témoignages dont 371 ont été consignés dans deux manuscrits conservés à Sienne ${ }^{6}$.

6 Ce jour-là, le 10 septembre, arrive à la barre, un témoin enregistré en $81^{\mathrm{e}}$ position dans l'ordre des consignations du manuscrit ${ }^{7}$. Comme les autres déposants, il décline son identité : Manfredinus domini Francisci; son lieu de résidence: Tolentino; donne son âge : quarante-cinq ans; et, devant les commissaires, en touchant la Bible (tactis sacrosanctis evangeliis) jure « de dire la vérité au sujet des articles, de la vie, des mœurs et des miracles de frère Nicolas de Tolentino ». Comme de nombreux témoins de ce procès, Manfredinus appartient à l'oligarchie urbaine. Noble de Tolentino, il possède des maisons dans les deux plus riches quartiers de la ville: dans un document contemporain, une riformanza datée de 1312, il apparaît parmi les vingt-cinq habitants les plus riches du quartier Saint-Catervo et, à la fin du mois d'octobre de cette même année (toujours selon le même document), il est chargé par la commune, avec sept autres personnes, de choisir les électeurs du bourg voisin d'Urbisaglia, en tant que représentant $d u$ quartier Sainte-Marie ${ }^{8}$. Selon des témoignages du procès, en 1310 , il habite dans ce dernier quartier. On le rencontre comme témoin instrumentaire et représentant de la ville dans divers actes concernant la commune dans les années qui 
précèdent l'enquête 9 . Il possède également une ou plusieurs maisons dans le village de Santa Lucia (à quelques kilomètres au nord-ouest de Tolentino) et de nombreux domestiques ${ }^{10}$.

7 À lire le procès-verbal, le 10 septembre 1325 , comme la veille, jour de l'arrivée de la commission à Tolentino, les commissaires semblent débordés par la foule de pèlerins venue dans la ville pour le $20^{\mathrm{e}}$ anniversaire de la mort de Nicolas : les deux évêques « ne pouvaient pas en entendre autant à cause de la foule innombrable de gens à examiner... $»^{11}$. Lors de ces deux journées d'audition, lorsqu'ils ont interrogé les témoins, il l'ont fait très rapidement; en témoigne la maigreur des dépositions enregistrées. Cependant, comme en rend compte le nombre d'occurrences de la transcription écrite de son témoignage, Manfredinus, lui, a été longuement entendu ${ }^{12}$. Il rapporte trois miracles. Intéressons-nous au dernier. Il raconte que domina Alixa (ou Anfelixia ou Lixa), sœur de son épouse Ymilia (sa cognata) et épouse de Mercatante Johannis Ade de Tolentino (voir document en annexe) est restée aveugle durant plus de trois ans avant d'invoquer longuement Nicolas afin qu'il intercède auprès de Dieu et qu'elle a été guérie un vendredi de mars 1306. À la question de savoir s'il connaissait Alixa avant sa maladie puis infirme puis guérie, Manfredinus répond « oui car c'était la sœur de son épouse $»^{13}$.

8 Originaire de Tolentino, Alixa, comme de nombreuses femmes de cette région, s'est mariée très jeune, vers seize ou dix-sept ans. Quittant son quartier (peut-être celui de Saint-Martin), elle va habiter in domo dicti Mercatantis dans le quartier le plus riche et le plus prestigieux de la ville, celui de Saint-Catervo. Mercatante est d'un rang légèrement inférieur à Manfredinus: on ne le trouve ni dans la liste des cent habitants les plus riches de Tolentino de 1312 ni dans le Conseil de la Credenza de la même année. Il n'en demeure pas moins d'un niveau social élevé : il possède une maison très vaste avec un cloître (claustrum) une domestique et, vers 1305, un épervier, symbole de condition noble. Alixa a vécu sana un peu plus d'un an après le miracle avant de mourir, au printemps 1307, à l'âge de vingt-et-un ans environ.

9 Six autres témoins ont rapporté le même prodige, avec quelques variantes. Frère Natimbene de San Severino (témoin 9), un ermite de Saint-Augustin qui, comme la grande majorité des témoins issus de l'ordre de Nicolas, n'a pas assisté à la scène mais le rapporte uniquement par ouïe dire (secundum quod audivit), témoigne le 23 juillet. Mancinus quondam Fortis (témoin 88) originaire de Sant'Angelo in Puntano (village natal de Nicolas) mais habitant de Tolentino en 1325, lui aussi, informé par d'autres, a fait un très court récit, le 30 juillet $^{14}$. Un notaire Jacobus magistri Johannis (témoin 80 ), autre membre de l'oligarchie urbain $\mathrm{e}^{15}$, qui tient son information très succincte de la miraculée, dépose également le 30 juillet. Jacobucia (témoin 83), sans doute une voisine et amie de la famille, est interrogée le 10 septembre. Domina Bruna Pensanicti (témoin 272), une autre voisine âgée de quarante-deux ans, témoigne le 31 juillet ; sa déposition est de très loin la plus riche ${ }^{16}$. Enfin, Mercatante (témoin 78), le mari d'Alixa, livre son témoignage le 29 juillet et peut-être également le 10 septembre.

D'autres personnes, qui n'ont pas témoigné ou dont la déposition n'a pas été retenue par les commissaires, sont mentionnées par les sept témoins à un moment ou à un autre du processus miraculeux. Le plus loquace à ce sujet est Mercatante, qui est davantage capable, près de vingt ans après les faits, de se souvenir de ceux et de celles qui étaient dans sa maison. Ces presentes ont parfois joué un rôle bien plus important auprès d'Alixa et auraient pu apporter davantage d'informations sur l'événement. Il 
s'agit de Gaudente Bonconsilii, d'Accula, une famula de la famille, de Plana uxor Canelli, de Flordalixa, belle-mère (mère de Mercatante) d'Alixa, des deux filles d'Alixa (Amorosa et Lisa), d'Ymilia sa sœur, citée à comparaître le 6 août à Tolentino et qui semble avoir témoigné (mais sa déposition n'a pas été consignée) ${ }^{17}$, de Manfredinus, son beau-frère (époux de Ymilia), d'Orsella, sa belle-sœur (sœur de Mercatante), de Raynalducius, son beau-frère (frère de Mercatante et d'Orsella) et de Rocius et Menaldus Gualterucii, deux autres frères d'Alixa et Ymilia ${ }^{18}$ (voir document en annexe). Rien ne permet de savoir si ces « présents » vivent à demeure, se sont installés dans la maison de Mercatante à cause de la cécité d'Alixa ou sont seulement de passage au moment du miracle ${ }^{19}$.

11 Il existe donc un écart sensible entre les personnes les plus présentes et actives au moment des faits et les témoins retenus. Dans ce cas, la sélection se réalise principalement aux dépens des femmes et des domestiques. Au sein d'une procédure inquisitoriale commandée par la papauté, le témoignage n'a pas pour fonction d'être le plus précis possible. Pour les commissaires, il ne s'agit pas de cette vérité que l'historien recherche mais d'une autre vérité, contrôlée par les Augustins et les notables de Tolentino, celle qui doit prouver la sainteté de Nicolas.

Le groupe d'entraide convoqué autour d'Alixa est composé de membres apparentés ou non. La résidence est poreuse et laisse entrer en son sein, des amis et des voisins. Dans ce cas cependant, les membres de la famille étroite jouent un rôle central. Comme Alixa, habite "dans la maison de son mari ", les affins se montrent très présents (Flordalixa, Orsella, Raynalducius). Mais, sans doute à cause d'une faible exogamie géographique (la délocalisation de l'épouse s'est réalisée d'un quartier à un autre), la famille d'origine (Ymillia et son époux Manfredinus mais aussi Rucius et Menaldus) tient une place non négligeable. Parmi les consanguins ou les affins, le lien adelphique est dominant puisque, au total, cinq frères et sœurs sont présents : une sœur et deux frères de l'épouse et un frère et une sœur du mari.

La présence plus forte de la fratrie de l'époux

13 L'écrasante majorité des parents rencontrés dans le procès de Nicolas de Tolentino appartient à la famille étroite. Sur un total de 306 miracles, auprès du témoin et/ou du miraculé (tout sexe confondu) à un moment ou un autre du processus miraculeux, ont pu être comptabilisés 92 mères, 52 pères, 29 frères, 20 sœurs, 24 épouses et 20 époux. Lorsque le miraculé est un enfant (garçon ou fille), les parents dominent très nettement et les femmes de la famille sont davantage présentes : 78 mères (sur 92) contre 41 pères (sur 52), 7 sœurs (sur 20) contre 1 frère (sur 29). Comme nous avions pu le montrer à partir d'autres sources hagiographiques, l'aînée joue toujours un rôle plus fort que le premier des garçons auprès de ses frères et sœurs en enfance, sorte de «mère de substitution $»^{20}$.

Lorsque le miraculé est adulte, en revanche, les frères et les sœurs ( $41: 28$ frères et 13 sœurs) sont nettement plus présents que les parents ( $25: 14$ mères et 11 pères) ou que les époux et les épouses. Si l'on tient compte d'une relative présence auprès du miraculé-enfant, des tantes (10) et des oncles (12), qui sont aussi les frères et les sœurs des parents (sans parler du rôle joué par les beaux-frères ou les belles sœurs, comme on peut l'observer dans le cas d'Alixa), on peut affirmer qu'au sein des réseaux de solidarités activés au moment de la maladie, de la mort, de l'accident ou du miracle, la relation adelphique est la plus importante.

15 La parenté du mari est plus fréquente que celle de l'épouse. On compte ainsi davantage d'oncles et de tantes paternelles que maternelles auprès des enfants miraculés (10 
oncles paternels pour 2 maternels et 7 tantes paternelles pour 3 maternelles). Les frères et les sœurs du mari sont également plus nombreux que ceux de l'épouse. Lors du miracle de Sennucia, la nonne Paulucia (témoin 29) explique qu'en 1305, avant de rentrer au monastère, elle habitait in domo fratris sui Manfredini domini Francesci ${ }^{21}$. À cette date, Manfredinus (le beau-frère de notre Alixa), âgé de vingt-cinq ans, est déjà chef de maison et père d'un enfant. Dans la ville voisine de Macerata, à la fin du XIV siècle, il n'est pas rare non plus de rencontrer un frère ou une sœur célibataire du mari au sein des noyaux familiaux ${ }^{22}$.

16 Mais, il arrive aussi qu'une femme célibataire ou veuve trouve refuge dans la maison de sa sœur mariée. Vers 1323, lorsque la petite Catalina souffre de l'estomac, c'est la sœur de sa mère, domina Aldisia Jacobucii (témoin 91), âgée de quarante-huit ans, qui se charge d'invoquer Nicolas, d'amener sa nièce au sanctuaire et qui la ramène guérie « dans la maison de sa sœur", sise dans le quartier de Saint-Catervo (malgré la présence des parents de l'enfant). À la question automatique (car faisant partie des forma interrogatorii de la procèdure inquisitoire relative au procès de canonisation) « si elle la \{Catalina\} connaissait ", en utilisant une formule qui ressemble fort à celle utilisée par Manfredinus pour Alixa ${ }^{23}$, "elle dit que oui car elle était, comme on le dit, sa nièce et qu'elle habitait dans cette maison avec sa sœur ${ }^{24}$ ».

17 Cette inégalité entre branche paternelle et maternelle ne se retrouve pas (sur un effectif certes beaucoup moins important) au niveau de la génération des grands parents : quatre grands-mères maternelles pour quatre grands-mères paternelles. Cette égalité et l'absence de grands-pères (des deux branches) est sans doute due à une différence d'âge au mariage élevée entre les conjoints (dix à douze ans de plus pour le mari) dans les Marches de la première moitié du XIv siècle $^{25}$.

L'explication par la virilocalité

18 Nous pensons que la plus forte présence des frères et des sœurs du mari dans la maison s'explique en grande partie par la domination du mariage virilocal ou patrilocal dans les Marches de la première moitié du XIV siècle.

Dans l'Italie communale, les structures de parenté et les modes de transmission des biens, en encourageant des liens forts entre les frères, favorisent largement la virilocalité ou la patrilocalité. En effet (et en cela il n'existe pas de rupture avec la noblesse foncière des siècles précédents), dans le monde communal italien domine le principe d'agnation qui empêche les éléments féminins de participer à la succession ou limite cette participation, retenant les biens parmi les descendants en ligne masculine ${ }^{26}$. S'ajoute à ce constat, du moins en Italie centrale, la faiblesse du système de la primogéniture et la fréquence des biens en indivision ${ }^{27}$ qu'attestent de nombreuses indications telle que filii $X$ ou heredes $X^{28}$. Dans le procès, le moulin où se déroule l'accident du petit Puccius appartient "aux fils de dominus Gentilis de San Ginesio ${ }^{29}$ ». Dans les archives du couvent de Tolentino, douze actes (rédigés entre 1307 et 1345) concernent la fratrie Petri Lanzoni, dont deux membres sont témoins (Esnudus, témoin 226, âgé de quarante ans, et son frère aîné Jacobus, témoin 227, âgé de cinq de plus, qui fait partie des vingt-cinq habitants les plus riches du quartier Sainte-Marie en 1312) ${ }^{30}$. La fratrie (représentée d'abord par l'aîné Jacobus, puis, à partir des années 1330, par Esnudus) très souvent en commun, acquiert de nombreux biens. La formule la plus fréquente rencontrée dans les actes est : « ... Domino Jacobo domini Petri Lançoni recipienti et ementi pro se et vice et nomine magistri Symonicti, Esnudi, Lancicti et Verendutii eius 
fratrum et eorum heredum... ». Esnudus, localise le miracle survenu en septembre 1315, dans une maison qui appartient à l'ensemble des frères ${ }^{31}$.

À la mort du père, les frères se retrouvent souvent copropriétaires d'une grande partie du patrimoine paternel et font fructifier ce dernier en achetant des biens ensemble, occasion de maintenir des liens (bons ou mauvais) et incitation à demeurer grouper et à " héberger » leurs épouses. Le principe de l'exclusio propter dotem, toujours très présents dans les statuti des Marches du début du XIv e siècle, ancre un peu plus les intérêts (économiques) de l'épouse dans sa famille d'orientation.

Dans le procès de canonisation de Nicolas de Tolentino, un certain nombre d'indices attestent cette virilocalité. D'une part, dans leur présentation ou lors de leur témoignage, certaines femmes distinguent soigneusement leur lieu d'origine, de mariage et de vie: domina Mita (témoin 127) explique qu'elle a entendu la fama de Nicolas «dans la terre (terra) de Treia où elle s'est mariée (nupta), dans celle de San Ginesio d'où elle est originaire (oriunda) et à Pollenza où elle vit (conversata est) ${ }^{32}$ ». Mita a donc parcouru une trentaine de kilomètres vers le Nord pour s'installer dans le village de son époux (magister Matheus de Treia) puis, sans que l'on sache la raison (veuvage ?), s'est déplacée à nouveau d'une dizaine de kilomètres vers le Sud pour s'installer à Pollenza (voir carte en annexe). D'autre part, de nombreuses femmes (bien plus que les hommes) sont présentées comme olim de tel lieu et nunc de tel autre. La distance géographique qui sépare olim de nunc permet souvent de mesurer l'exogamie. Enfin, interrogées sur le lieu de la maladie, de l'invocation ou du miracle (interrogata de loco...), les femmes répondent majoritairement in domo sua patris ou in domo sua mariti (ou viri). À la même question, les hommes répondent toujours (où le notaire a écrit) : in domo ipse testis ou in domo sua propria. Ainsi, lorsque magister Jacobus magistri Johannis (témoin 80 ) rapporte le miracle de cécité d'Alixa et qu'on lui demande le lieu de l'invocation, il répond « dans la maison du mari de cette dame ${ }^{33}$ ». À la même question, la voisine et amie Jocobucia (témoin 88) répond « dans la maison de Mercatante, le mari de la dite domina Anfelixia ${ }^{34} »$. Tout se passe comme si la femme (ou le notaire qui transcrit son témoignage) ne pouvait pas s'approprier son lieu de vie, malgré son intégration à la famille par alliance beaucoup plus solide qu'en Toscane ${ }^{35}$. La veuve, contrairement à ce qu'on observe dans cette dernière région à la même époque, reste très attachée au lignage du mari, aidée en cela, par les coutumes successorales : ainsi les statuts de Macerata défendent le patrimoine agnatique contre les tentatives de récupération de la famille de la veuve plus efficacement qu'en Toscane alors qu'au contraire le veuf peut conserver une part très importante de la dot pour la transmettre à ses héritiers ${ }^{36}$.

Dans les actes notariés, l'on peut peut-être percevoir cette virilocalité au moment des contrats de mariage à l'utilisation de l'expression uxorem ducere, préféré à uxorem accipere. La première locution semble se référer plus spécifiquement à la deductio de l'épouse dans la maison de son mari, tandis que la seconde (accipere) exprime davantage la bilatéralité ${ }^{37}$.

D'autres documents de la région attestent cette virilocalité. Certains traités de paix comportent des clauses obligeant les communes à s'échanger des jeunes filles en mariage. Ainsi, le 18 février 1306, lorsque Camerino conclut la paix avec Matelica, San Severino et Fabriano, une clause stipule qu'avant six mois, quarante demoiselles de diverses conditions sociales de Camerino devront se marier à San Severino, vingt à Matelica et dix à Fabriano. En échange (vice versa) les hommes de Camerino s'engagent 
à prendre pour épouses un nombre équivalent de femmes originaires des trois villes. Les nouveaux conjoints bénéficieront de la complète citoyenneté dans leur nouveau lieu de résidence ${ }^{38}$.

Une virilocalité à faible distance : problèmes de définitions

24 L'ensemble de ces exemples prouve la nette domination des mariages virilocaux. Cependant, ils attestent également que les jeunes mariées parcourent une faible distance (ne dépassent guère le "pays de connaissance ») pour s'installer dans leur nouveau lieu de résidence, même dans les milieux dirigeants des quasi-città des Marches au début du XIV ${ }^{\mathrm{e}}$ siècle. La Marche d'Ancône présente une structure urbaine originale que Bartolo de Sassoferrato (1314-1357), auteur vers 1330 d'un Tractatus de Regimine civitatis, définit comme une "provincia castellorum», une région peu urbanisée, constellée de très nombreux castelli ou bourgs perchés, très proches les uns des autres et de quasi-città, structure qui favorise un isolat restreint.

Ces échanges matrimoniaux se déroulent même souvent, comme nous venons de le voir, au sein d'une même ville, d'un même village ou d'un même quartier, posant à l'historien un problème de définition. Nous qualifions, en effet, ce type d'union de virilocal, même si, comme dans le cas des deux sœurs, Alixa et Ymilia, il s'agit d'une exogamie de quartier ou d'une endogamie de castrum (de Saint-Martin, à Saint-Catervo pour l'une et à Sainte-Marie pour l'autre). Il serait nécessaire que les historiens se dotent d'un vocabulaire plus fin pour qualifier ces mariages en tenant compte de la distance géographique de délocalisation et de la configuration de la nouvelle demeure. Car, il est toujours difficile de savoir si la nouvelle épouse part habiter avec son mari dans une maison nouvelle (mariage virilocal) ou si elle s'installe chez le père de son époux (mariage patrilocal) en conformité avec le principe patrilinéaire qui domine dans la société italienne de la fin du Moyen Âge. Le flou et parfois la confusion des définitions données par les anthropologues et les historiens prouvent la difficulté d'approche du phénomène. Ainsi, la patrilocalité est définie comme «la résidence des nouveaux époux avec ou auprès des parents de l'époux ${ }^{39}$ ». Pour qualifier les unions matrimoniales dans les Marches du début du XIv ${ }^{e}$ siècle, le avec et le auprès ne peuvent être mis en synonymie. Pour l'anthropologue Robert Deliège, il y a résidence virilocale "lorsque le jeune couple s'installe dans la famille ou le village du jeune homme » et résidence uxorilocale «lorsque le couple s'installe chez la jeune fille $»^{40}$. L'absence de symétrie des définitions, souvent adoptée par les historiens, confirme cette confusion.

Pour démontrer la difficulté d'approche et de définition, prenons l'exemple de Berardescha et de Ceccha, les deux filles de Berardus Appilaterre, notable le plus en vue du procès, habitant le quartier Saint-Catervo, celui qui a su, plus que les autres, instrumentaliser l'enquête de 1325 à son profit. L'aînée, Berardescha (vingt-cinq ans en 1325), se marie en septembre 1314, à l'âge de quatorze ans avec Anthonius Thomaxii de Parisinis, notarius et mercator de Tolentino. Le nouveau couple part habiter chez le père d'Anthonius dans le quartier Saint-Jean (mariage patrilocal). Quelques années plus tard, la cadette, Ceccha (vingt-trois ans), à la suite de son mariage, va résider avec son nouvel époux dans une maison différente de celle de son beau-père : in domo propria viri sui ${ }^{41}$ (mariage virilocal ou encore néolocal dans la mesure où les nouveaux époux élisent domicile dans un lieu différent de celui où l'un et l'autre vivaient avant l'union matrimoniale). Mais, cette nouvelle demeure se situe dans le même quartier, à quelques maisons des parents de Ceccha. Cette dernière se déplace donc un peu moins que sa sœur aînée. Il s'agit certes d'une virilocalité mais on pourrait aussi valider le terme de 
matrilocalité définie comme le fait de s'installer auprès des parents de l'épouse. Le destin matrimonial de ces deux sœurs ressemble fort à celui, déjà évoqué, d'Ymillia et d'Alixa.

Pour qualifier les unions matrimoniales des notables de l'Italie centrale, Jean-Claude Maire Vigueur a proposé d'utiliser le terme de "viripatrilocalité » car, dans les cas qu'il étudie, il existe des blocs lignagers, souvent regroupés dans des "quartiers lignagers ", au centre des villes. La résidence nobiliaire possède une structure interne qui doit répondre à la nécessité pour le père de famille de fournir à ses fils qui se marient une maison autonome qui soit la plus proche possible de la sienne. Cette habitation, distincte de celle du chef de famille, peut se réaliser parfois au prix d'aménagements du bloc (cloison, galerie de raccordement). Pour mettre en application ce principe, il faut des moyens : posséder un groupe de maisons permettant d'assurer une «continuité topographique du lignage ${ }^{42} »$.

Afin de pouvoir qualifier ces unions matrimoniales, on le voit, les deux notions clés à prendre en compte sont le lieu de résidence et la distance accomplie par l'épouse. Pour la majorité des acteurs sociaux et pour les dirigeants, c'est le fait d'habiter, de vivre sous le même toit qui est capital. Pour les autorités communales de Macerata au tournant des $\mathrm{XIV}^{\mathrm{e}}-\mathrm{XV}^{\mathrm{e}}$ siècles, le couple ne devient un feu fiscal que lorsqu'il est distinct de celui du père: "l'unité géographique du foyer, la maison l'emporte sur le lien familial et le degré de parenté43 $»$. La question centrale qui est posée est celle de savoir, à partir de quel critère peut-on considérer qu'il n'y a pas changement de lieu ? Même maison? Même «bloc lignager »? Même quartier? Même castrum ? Il serait donc impératif que les historiens de la parenté s'accordent sur une terminologie plus précise et plus en phase avec la réalité pour éviter de parler un langage différent.

Quoi qu'il en soit, il est évident que le plus ou moins fort maintien du lien entre l'épouse et sa famille d'origine (ses frères et ses sœurs en particulier) est conditionné en partie par cette distance de délocalisation.

La résistance du lien adelphique à la virilocalité

30 C'est en grande partie cette faible délocalisation consécutive au mariage dans les Marches de la première moitié du XIV ${ }^{\mathrm{e}}$ siècle qui amenuise la dissension des liens adelphiques ou évite leur rupture et explique le nombre important de frères et de sœurs de l'épouse auprès du miraculé ou de ses parents. Les événements forts de la vie des femmes, comme l'accouchement, sont des instants propices à saisir le « retour des sœurs ». Dans le procès, c'est souvent au moment des parturitions que l'on voit les mères et les sœurs de l'épouse présentes dans la maison (du mari). Les statuti de la région légifèrent pour limiter le nombre de femmes venant rendre visite à l'accouchée. En 1322, ceux de Camporontondo interdisent aux femmes d'aller voir une mulier infantata ou une mulier pariens sauf si elles sont «mater, soror carnalis et consobrina et cognata (et consanguinea) vel consobrina... ${ }^{44} \gg$. L'ordre des membres de la parenté autorisés dessine divers cercles de solidarité : la famille étroite en priorité (mère et sœur), puis la nièce ou la cousine germaine (première mention de consobrina) puis les belles-sœurs, consanguines ou cousines (seconde mention de consobrina). Cette solidarité se manifeste aussi au moment où la femme est battue ou lorsque les relations conjugales sont distendues ${ }^{45}$.

31 La trajectoire des legs testamentaires (comme l'élection de sépulture de l'épouse) atteste également le maintien des liens avec la famille d'origine au moment de la mort. Dans son testament, daté du 27 mai 1340, domina Grana Sinibaldi de Tolentino, veuve et 
remariée à magister Corradus Petri, n'oublie ni sa famille d'origine, ni sa première famille d'orientation. Elle ne lègue qu'à des femmes de sa parenté : deux nièces : Rosa (fille de son frère, Corradus Sinibaldi) et Margarita ; sa sœur Mathia ; et son ex-belle-mère, Facultia (mère de Nicolutius, quodam viri sui). Elle rappelle que son second mari, Corradus Petri, avait déjà fait un testament dans lequel il donnait une terre au couvent des Augustins de Tolentino, sise dans la contrata de Remutulus pour dresser un autel. Elle demande à ce qu'on y célèbre des messes pro anima sua et pro animabus predecessorum virorum suorum, associant dans la mort ses deux époux ${ }^{46}$.

Le procès et les actes de la pratique permettent donc d'observer ce que les anthropologues et les historiens du droit, spécialistes de l'Italie de la fin de l'époque médiévale constatent sur le plan juridique : la femme, « sauf en de très rares exceptions comme dans la Chine ancienne, n'est pas détachée entièrement de son groupe natal et absorbée dans le groupe, clan, lignage, ou famille qui a aliéné pour elle une part de ses richesses. Les femmes continuent d'avoir des droits au sein de leur groupe natal, et des obligations vis-à-vis de leurs membres ${ }^{47}$. " Thomas Kuehn remarque certes que dans l'Italie de la Renaissance, le mariage est « un double processus de séparation et de transfert du contrôle sur la femme et la propriété de sa famille d'origine à sa famille marital ${ }^{48} »$. Mais, poursuit-il, ces transferts ne signifient pas que la femme ne maintient pas de relations avec sa famille de naissance sur un plan juridique. De Martino (mort vers 1166) à Filippo Decio (mort en 1535), en passant par Carlo di Tocco (vers 1200) ou Bartolo da Sassoferrato (mort en 1357), les juristes reconnaissent que le mariage n'élimine pas complètement la patria potestas ${ }^{49}$. L'épouse des Marches, sur un plan juridique et sur un plan affectif, même devenue mère, demeure toujours une fille et une sœur ${ }^{50}$.

Le procès de canonisation de Nicolas de Tolentino et d'autres sources contemporaines de la pratique permettent donc de montrer le poids des liens adelphiques dans la vie quotidienne des individus. Ces liens, non seulement sont construits dès l'enfance en fonction des différences d'âge et de sexe, du rang dans la fratrie et du sexe de celle-ci, mais évoluent au cours du cycle de vie des individus selon qu'il y a poursuite ou non d'intérêts communs, proximité ou éloignement géographique. Pour la femme, il semble que ce dernier critère soit essentiel puisque, dans la grande majorité des cas, son mariage entraîne une plus ou moins grande délocalisation et, par conséquent crée une distance géographique avec sa famille d'origine. La « résistance manifeste (...) à ouvrir la cellule familiale de base aux parents par alliance ${ }^{51}$ ", qui semble caractérisée les formes du mariage en Italie centrale, n'empêche pas le maintien de fortes solidarités adelphiques auprès de l'épouse.

L'ethnographie des relations de parenté proposée à partir d'un procès de canonisation a permis de souligner que, parmi les acteurs et les actrices adultes, les liens d'alliance et de filiation comptent moins que les liens de germanité ou que les liens de familiarité. Afin de ne pas se limiter à décrire des structures sociales et des normes de comportement éloignées du vécu des individus, l'anthropologie de la parenté a tout intérêt à accueillir ce type de démarche empirique qui, en réduisant l'unité d'observation à des événements et des interactions, met au jour les attitudes effectives des membres de la parenté dans un contexte donné. 


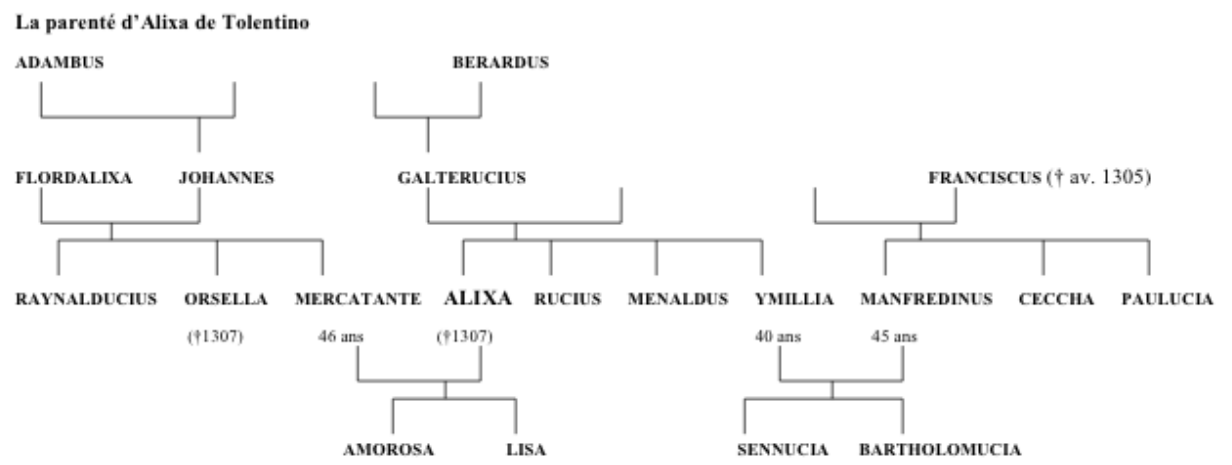

N.B. L'àge donné est celui de 1325

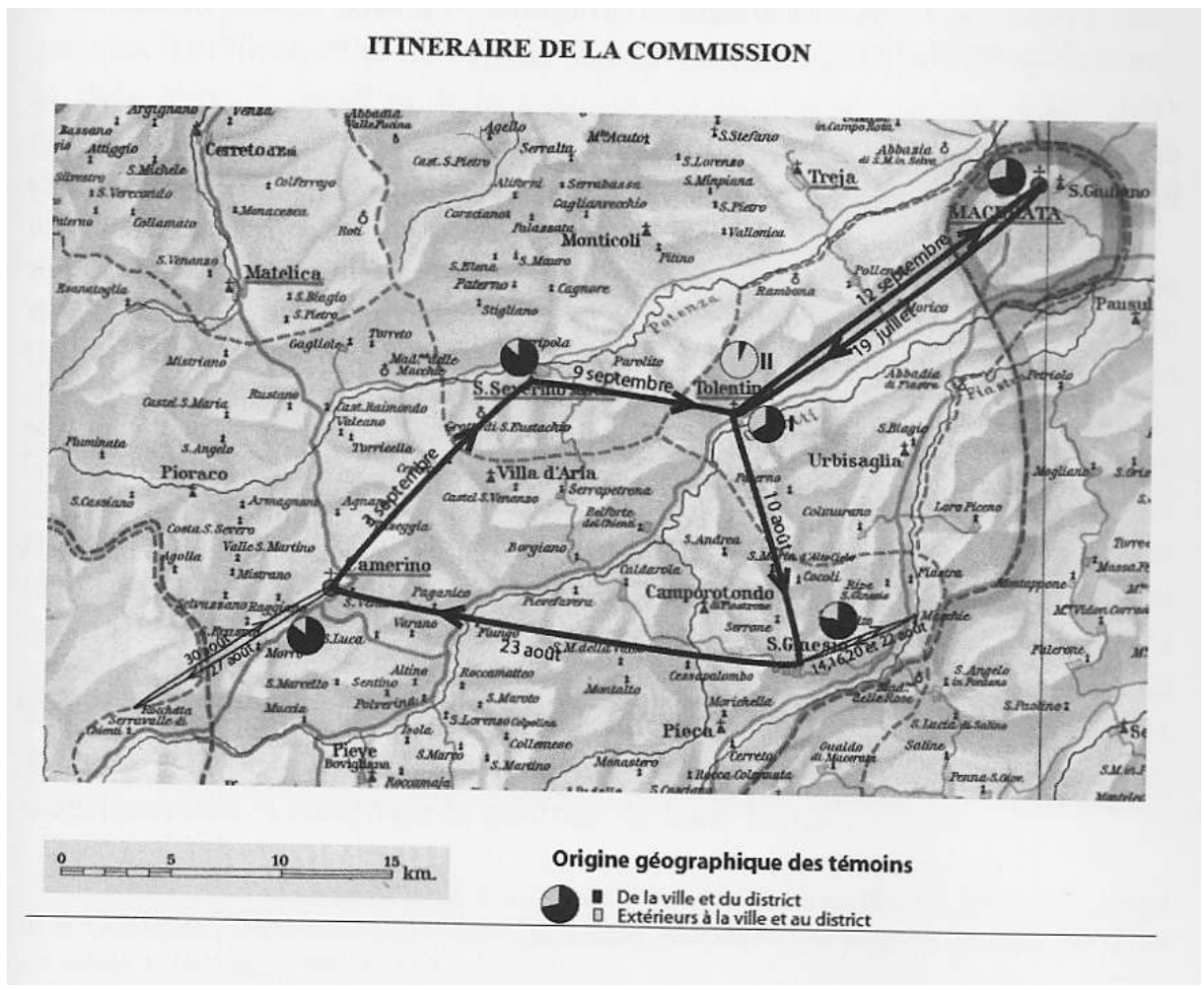

\section{NOTES}

1.F. WEBER, Le Sang, le Nom, le Quotidien. Une sociologie de la parenté pratique, Paris, 2005 ; voir aussi M. GODELIER, Métamorphoses de la parenté, Paris, 2004, p. 12.

2.J.-F. CHAUVARD et C. LEBEAU, « Introduction » à Éloignement géographique et cohésion familiale (XVe-XXe siècle), J.-F. CHAUVARD et C. LEBEAU, dir., Strasbourg, 2006, p. 7-8.

3. Comme on devrait davantage étudier cette capacité de résistance au moment de la mort d'un membre de la parenté ou lorsque les intérêts communs à l'intérieur d'un groupe s'altèrent ou prennent fin. 
4.Pour ne s'en tenir qu'aux ouvrages, voir, par exemple, R. C. FINUCANE, Miracles and Pilgrims. Popular Beliefs in Medieval England, 1977, Londres, rééd., 1995 ; M. GOODICH, Violence and Miracle in the Fourteenth Century. Private Grief and Public Salvation, Chicago et Londres, 1995 et D. LETT, Un procès de canonisation au Moyen Âge. Essai d'histoire sociale. Nicolas de Tolentino, 1325, Paris, 2008.

5.On lira avec profit, S. K. COHN, « Marriage in the mountains : the Florentine territorial state, 1348-1500", dans Marriage in Italy, 1300-1650, T. DEAN et K. J. P. LOWE dir, Cambridge, 1998, p. 174-196 et H. BROISE et J.-C. MAIRE VIGUEUR, « Strutture famigliari, spazio domestico e architettura civile a Roma alla fine del Medioevo » dans Storia dell'arte italiana, Parte 3, vol. 5, Momenti di architettura, Turin, 1983, en particulier p. 133-141.

6.Ils ont fait l'objet d'une édition en 1984 : Il Processo per la canonizzazione di S. Nicola da Tolentino, édition critique de N. OCCHIONI, Rome, 1984 (abrégé dans la suite de cet article en Procès).

7.Procès, p. 223-228.

8.Il est mentionné dans la liste des nomina centum hominum maioris appretii (vingt-cinq par quartier), Archivio storico del comune di Tolentino (désormais abrégé ACT), Fondo riformanze, $\mathrm{n}^{\circ} 1$, fol. 2 et représentant de la commune, Ibid., fol. 30. Dans la fiche d'identité du procès, comme dans la riformanza de treize ans antérieure, il ne porte pas le titre de noble. Pourtant, au cours du récit relatant la mort puis la résurrection de sa fille, le couple qu'il forme avec Ymillia (témoin 100) est qualifié de « noble ».

9.Présent comme témoin instrumentaire dans ACT du 26 avril 1313, 28 avril 1313, 16 mai 1314 et 12 février 1315.

10.Procès, p. 225.

11.Procès, p. 62. Nous avons montré qu'il existait une contradiction radicale entre cette liesse populaire constatée dans le procès et l'absence totale de mentions de Nicolas, en dehors des pièces relatives à son procès de canonisation, avant les années 1340, D. LETT, Un procès de canonisation... op. cit., p. 51-65.

12.Son témoignage compte 1590 mots (ce qui en fait le $24^{\mathrm{e}}$ plus long du procès) ; 44 témoignages seulement comptent plus de 1000 mots. Les 371 dépositions varient de 5891 à 27 mots. La moyenne est de 548 mots.

13.Procès, p. 226.

14.Dans le procès, être membre du même ordre que Nicolas ou être originaire du même village donne plus facilement « voix au chapitre », même lorsqu'on a peu de choses à dire sur un miracle auquel on n'a pas assisté.

15.Peut-être le $3^{\mathrm{e}}$ de la liste des vingt-cinq habitants les plus riches du quartier de Saint-Martin en 1312, ACT, Fondo riformanze, $\mathrm{n}^{\circ} 1$, fol. 3 et sans doute le frère d'un médecin de Tolentino : magister Petrus magistri Johannis. 16.Lorsque l'historien éclaire la parenté pratique et les solidarités familiales dans la société médiévale, il rencontre de nombreux voisins, amis ou « familiers », qui jouent parfois un rôle bien plus important auprès d'ego que les membres de la parenté.

17.Procès, p. 36.

18. Natimbene (témoin 9) indique que le père d'Alixa se nomme Gualterucius domini Berardi, Procès, p. 60.

19. Giovanni Cherubini pense que la mère, la sœur et le frère de Mercatante sont des « habitants de la maison ", G. CHERUBINI, Gente del medioevo, Florence, 1995, p. 26. La lecture du procès ne nous permet pas de valider cette hypothèse. Un certain nombre d'indices permettent uniquement de penser que Flordalixa (sans doute déjà veuve en 1306), mère de Mercatante, a élu domicile dans cette grande maison.

20.D. LETT, « La sorella maggiore 'madre sostitutiva' nei miraculi di san Luigi », dans Fratello e sorella, numéro spécial de Quaderni Storici 83, A. ARRU et S. BOESCH-GAJANO dir., n², août 1993, p. 341-353.

21.Procès, p. 150.

22.Ph. JANSEN, Démographie et société dans les Marches à la fin du Moyen Âge. Macerata aux XIVe et XV siècles, Rome, 2001, p. 206 et p. 220.

23. Voir supra, p. 56 
24.Procès, p. 266-267.

25. Sur cette différence d'âge au mariage, je me permets de renvoyer à D. LETT, « Il matrimonio e la coppia nelle Marche all'inizio del Trecento ", dans Santità e società civile nel Medioevo. Esperienze storiche della santità agostiniana, Tolentino, Biblioteca Egidiana, 2005, p. 58-60.

26.Voir, par exemple, P. CAMMAROSANO, « Les structures familiales dans les villes de l'Italie communale, $\mathrm{XII}^{\mathrm{e}}$-XIV ${ }^{\mathrm{e}}$ siècles », dans Famille et parenté dans l'Occident médiéval, G. DUBY et J. LE GOFF dir., Rome, 1977, p. 181-194.

27.J.C. MAIRE VIGUEUR, Cavaliers et Citoyens. Guerre, conflits et société dans l'Italie communale, XII ${ }^{-}$-XIII ${ }^{e}$ siècles, Paris, 2003, p. 291-293.

28.Voir quelques exemples dans D. HERLIHY, « Family solidarity in Medieval Italian History », dans Economy, Society, and Government in Medieval Italy. Essays in Memory of Robert L. Reynolds, D. HERLIHY, R. S. LOPEZ et V. SLESSAREV, dir., Kent, 1969, p. 177-178.

29.Procès, p. 133.

30. Les actes sont édités dans E. RUGGERI, Le piu' antiche pergamene dell'archivio del convento di San Nicola a Tolentino (1235-1345) (tesi di laurea, Rino Avesani dir.), Università degli studi di Macerata, 1972-1973 : actes 9 (1307), 12 (1309), 21 (1313), 26 (1320), 28 (1331), 30 (1334), 32 (1337), 45 (1343), 47 (1343), 48 (1343), 49 (1344) et 51 (1345). Jacobus est $17^{\mathrm{e}}$ de la liste de 1312 pour le quartier Sainte-Marie, ACT, Fondo riformanze, $\mathrm{n}^{\circ} 1$, fol. 2 . Le fait que ces douze documents aient été déposés dans le fonds des archives du couvent, atteste que la famille Lanzoni entretenait des relations privilégiées avec les augustins de Tolentino (l'un de ses membres est peutêtre devenu augustin, emportant ces actes avec lui).

31.In domo dicti testis et fratrum suorum (sic) posita in quarterio Sancte Marie, Procès, p. 502.

32.Procès, p. 331.

33.Procès, p. 223.

34.Procès, p. 233.

35. Région où Christiane Klapisch-Zuber juge les épouses dans la casa de leur mari comme des « visiteuses de passages » à l'« appartenance flottante », C. KLAPISCH-ZUBER, La maison et le nom. Stratégies et rituels dans l'Italie de la Renaissance, Paris, 1990, p. 64.

36.Ph. JANSEN, Démographie et société... op. cit., p. 221.

37.A. MARONGIU, Matrimonio e famiglia nell'Italia meridionale (sec. VIII-XIII), Bari, 1976, note 2, p. 13 ; l'auteur cite des expressions telles que ad domum ducere (datant de 1191) et apud se uxorem ducere (1232).

38.C. ACQUACOTTA, Lapidi e documenti alle memorie di Matelica raccolte ed ordinate d'all arciprete Camillo Acquacotta, Ancône, 1838, vol. 1 p. 111. Le texte est édité, vol. 2, 1839, doc. 100, p. 181-190 (l'extrait concernant ces mariages croisés se trouvent aux pages 184-185).

39. Histoire de la Famille, A. BURGUIÈRE, C. KLAPISCH-ZUBER, M. SEGALEN et F. ZONABEND dir., Tome 1, Mondes lointains, mondes anciens, Paris, 1986, glossaire, p. 631.

40.R. DELIÈGE, op. cit., p. 13.

41.Procès, p. 243.

42.J.-C. MAIRE VIGUEUR, Cavaliers et Citoyens... op. cit., p. 285-296. À la fin du Moyen Âge, les familles aisées de Rome sont plus promptes à transgresser la norme de l'endogamie territoriale qu'à se résigner à faire un mariage socialement déséquilibré ; c'est grâce à cette endogamie territoriale que les jeunes couples ont pu s'installer dans une maison appartenant soit au père de l'époux (dans un quartier lignagier) ou dans une partie de la grande domus paternelle, moyennant, le cas échéant, quelques réaménagements, soit dans une maison du père de l'épouse sans déroger à la règle viripatrilocale et suppléer ainsi à la carence du stock immobilier du père de l'époux, H. BROISE et J.-C. MAIRE VIGUEUR, « Strutture famigliari... », loc. cit., en particulier p. 140. En revanche, les auteurs pensent que l'exogamie est le moyen le plus utilisé pour les secondes noces afin d'éviter l'hostilité de la parenté et des voisins, ibid., p. 144. 
43.Ph. JANSEN, Démographie et société... op. cit., p. 202.

44. Statuta Castri Campirotundi (1322/1366), D. CECCHI éd., Milan, 1966, titre XXII, mulierem infantatam, p. 236.

45. Comme à Venise à la même époque où, en cas de difficulté à l'intérieur du couple, la femme trouve souvent un soutien au sein de sa famille d'origine, surtout auprès de ses frères, L. GUZZETTI, « Separations and separated couples in fourteenth-century Venice » dans Marriage in Italy... op. cit., p. 262.

46. Acte édité dans E. RUGGERI, Le piu' antiche... op. cit., acte 35, p. 108-111.

47.M. GODELIER, op. cit., p. 164. C'est une des raisons qui incite Maurice Godelier à critiquer la fameuse formule de Claude Lévi-Strauss : « la parenté repose sur l'échange des femmes par les hommes et pour les hommes ». Selon lui, le versement d'une dot ne signifie pas l'achat d'une femme. Sur cette remise en cause et les perspectives ouvertes en histoire médiévale, voir Laurent FELLER, « "Morgengabe”, dot, tertia : rapport introductif », dans F. BOUGARD, L. FELLER et R. LE JAN dir., Dots et douaires dans le haut Moyen Âge, (Collection de l'Ecole française de Rome-295), Rome, 2002, p. 1-25.

48.Th. KUEHN, « Women, marriage and patria potestas in late medieval Florence » dans ID. dir., Law, Family and Women. Toward a legal anthropology of Renaissance Italy, Chicago et Londres, 1991, p. 198.

49.Ibid., p. 200-201

50.Sur cette pluralité des identités et des statuts, je me permets de renvoyer à D. LETT, « Les mères demeurent des filles et des sœurs. Les statuts familiaux des femmes dans les Marches au début du XIV siècle », dans La Mère, A. PARAVICINI BAGLIANI dir., Florences, (Micrologus's Library-14), 2007.

51.Ch. KLAPISCH-ZUBER, « Déclin démographique et structure du ménage. L'exemple de Prato, fin XIV ${ }^{\mathrm{e}}$-fin $\mathrm{XV}^{\mathrm{e}}$ siècle ", dans Famille et parenté..., op. cit., p. 263-264.

\section{RÉSUMÉS}

La documentation marchésane du début $\mathrm{du}$ XIV $\mathrm{v}^{\mathrm{e}}$ siècle (essentiellement le procès de canonisation de Nicolas de Tolentino daté de 1325) permet de mettre en évidence la fréquence du mariage virilocal (ou patrilocal). Elle autorise également à étudier les conséquences de l'éloignement géographique de la femme de sa famille de naissance sur les relations entre frères et sœurs. On observe que vivre sous le même toit ou appartenir à un même micro-espace entraîne des pratiques familiales et des interactions très différentes entre acteurs car les sentiments sont conditionnés par le partage quotidien d'expériences, des intérêts communs et des échanges. Dans les dépositions réalisées lors du procès de canonisation, on constate également la forte présence des frères et sœurs auprès du témoin et/ou du miraculé. Mais, à cause, de la prégnance du mariage virilocal, les frères et sœurs du mari sont davantage présents que ceux de l'épouse. Cependant, les liens adelphiques de la femme résistent, en partie à cause d'une délocalisation à faible distance.

Sibship links and geographical endogamy in early fourteenth-century Marche. The marchesan documentation of the early fourteenth century (especially the canonization process of Nicholas of Tolentino in 1325) allows us to highlight the frequency of virilocal (or patrilocal) marriage. It allows us also to study the consequences of a woman's geographical distance from her family of birth on the relations between brothers and sisters. One can notice that to live under the same roof or to belong to the same micro-environment involves very different family practices and interactions between agents because the feelings of the people are conditioned by the fact of 
sharing day after day experiences, common interests and relationships. In the depositions made in the canonization process, one can also note the strong presence of brothers and sisters near the witness and/or the person touched by a miracle. But, because of the importance of virilocal marriage, a husbands's brothers and sisters are more present than those of the wife. However, a woman's adelphic links resist, in part because her displacement is usually limited to a short distance.

\section{INDEX}

Mots-clés : frères, Marches (Italie), mariage, procès de canonisation, sœurs, virilocalité

Keywords : brothers, canonization process, Marche (Italy), marriage, sisters, virilocality

\section{AUTEUR}

\section{DIDIER LETT}

Université Paris I-Panthéon-Sorbonne - LAMOP, UMR 8589, 17 rue de la Sorbonne, 75005 Paris 